\title{
SAND97-0489C \\ CONF-97/14--1 \\ The Effect of Algorithm-Agile Encryption on ATM Quality of Service
}

\author{
Peter Sholander, Thomas Tarman, Lyndon Pierson, Robert Hutchinson \\ Sandia National Laboratories, P.O. Box 5800, Albuquerque, NM 87185 \\ email: peshola@sandia.gov
}

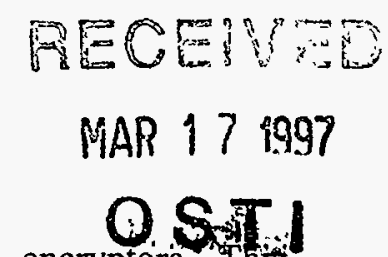

ABSTRACT:

Asynchronous Transfer Mode (ATM) users often open multiple ATM Virtual Circuits (VCs) to multiple ATM users on multiple ATM networks. Each network and user may implement a different encryption policy. Hence ATM users may need shared, flexible hardware-based encryption that supports multiple encryption algorithms for multiple concurrent ATM users and VCs. An algorithm-agile encryption architecture, that uses multiple, parallel encryption-pipelines, is proposed. That algorithm-agile encryptor's effect on the ATM Quality of Service (QoS) metrics, such as Cell Transfer Delay (CTD) and Cell Delay Variation (CDV), is analyzed. Bounds on the maximum $C D V$ and the $C D V$ 's probability density are derived.

\subsection{INTRODUCTION:}

The Phase 1 ATM Forum Security Standard specifies, amongst other things, end-to-end network encryption for an ATM Virtual Circuit (VC) [1]. In a distributed computing environment, an ATM user may open multiple ATM VCs to multiple ATM users on multiple different ATM networks. Each ATM user/network may implement a different encryption policy. High-speed ATM links are typically shared by several ATM users. For these reasons, ATM users may want access to shared, flexible encryption that supports multiple concurrent ATM VCs and multiple concurrent ATM users. Software-based encryption is flexible, but it sometimes raises performance and security concerns. Hence, some users require hardware-based encryption. Key-agile encryptors are the current state-of-the-art in high-speed, shared hardware-based ATM encryptors [2,3].

A key-agile encryptor uses the same encryption algorithm, with the same key length, for each VC. However, the encryptor maintains separate end-to-end encryption-contexts and encryption keys for each VC. This permits sharing by one or more ATM users. However, since a large user-community may use more than one encryption algorithm, each ATM user might need

This work was supported by the U.S. Department of Energy under Contract DE-AC04-94AL85000. access to several different key-agile encryptors. This raises both cost and administrative issues.

An algorithm-agile encryptor would allow each $\mathrm{VC}$ to use a different encryption algorithm. Of course, an algorithm-agile encryptor still has to maintain separate end-to-end encryption contexts and encryption keys for each VC. However, a single algorithm-agile encryptor will likely provide both initial-cost savings and administrativecost savings over several key-agile encryptors. Algorithmagile encryptors may also simplify connection setup if security policy becomes a routing metric within protocols such as ATM's PNNI (Private Network-Network Interface).

The context-switching time between the different VCs' encryption-contexts can limit the performance of both key-agile and algorithm-agile encryptors. Ideally, the context-switching mechanism would sustain rates between 106,132 contexts/sec (for $45 \mathrm{Mb} / \mathrm{s} \mathrm{DS} 3$ links) and $5,660,377$ contexts/sec (for $2.4 \mathrm{~Gb} / \mathrm{s}$ SONET OC-48 links).

For algorithm-agile encryptors, there are additional performance limitations. First, if the algorithmagile encryptor uses a single shared-processor then the context-switching time between the different encryption algorithms may further limit performance. Preliminary results indicate that the switching time between different encryption algorithms may be much larger than the switching time between different VC's encryptioncontexts. (For example, the key-switching and algorithmswitching times are $60 \mathrm{~ns}$ and $61 \mathrm{~ms}$, respectively, for Altera $10 \mathrm{~K} 100$ complex programmable logic devices.) Hence, a single, shared-processor architecture may not scale, to Gb/s ATM link rates. Alternatively, an algorithmagile encryptor could use multiple parallel-processors. Each processor could then execute a different encryption algorithm. During call-setup, a VC would request a given encryption algorithm. The call-request would proceed if either: a) that algorithm is already loaded in one of the multiple processors, or b) there is an idle processor that can load the requested algorithm. In this scenario, $M$ processors could support $\mathrm{N}$ algorithms where $\mathrm{N}$ may be greater than $\mathrm{M}$. This may provide a cost saving over $\mathrm{N}$ key-agile encryptors. This multi-processor architecture

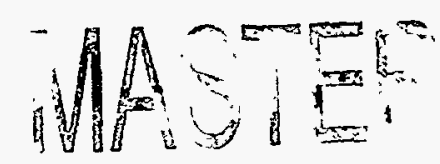




\section{DISCLAMIER}

Portions of this document may be illegible in electronic image products. Images are produced from the best available original document. 


\section{DISCLATMER}

This report was prepared as an account of work sponsored by an agency of the United States Government. Neither the United States Government nor any agency thereof, nor any of their employees, make any warranty, express or implied, or assumes any legal liability or responsibility for the accuracy, completeness, or usefulness of any information, apparatus, product, or process disclosed, or represents that its use would not infringe privately owned rights. Reference herein to any specific commercial product, process, or service by trade name, trademark, manufacturer, or otherwise does not necessarily constitute or imply its endorsement, recommendation, or favoring by the United States Government or any agency thereof. The views and opinions of authors expressed herein do not necessarily state or reflect those of the United States Government or any agency thereof. 
might scale to $\mathrm{Gb} / \mathrm{s}$ ATM link rates. However, callrejection can occur because of "algorithm-blocking", during call-setup, if $N>M$.

A second problem, with algorithm-agile encryption, is its effect on the ATM Quality of Service (QoS). (In brief, there are three important ATM QoS metrics -- namely cell loss, Cell Transfer Delay (CTD) and Cell Delay Variation (CDV). In [4], the CDV is defined as the difference between the best-case and worst-case CTD, where the best-case is the fixed network-delay and the worst-case is the CTD likely to be exceeded with a userdefined probability $\alpha$. "Excessive" CTD is undesirable for applications, such as voice, web-browsing and interactive game-playing, that have response-time requirements. "Excessive" CDV is undesirable since it complicates timing recovery for Constant Bit-Rate (CBR) applications such as video. CDV can also cause buffer overflows, and hence cell-loss. The ATM network and the ATM user negotiate mutually acceptable QoS values, for a particular VC, during call setup.) Each encryption algorithm, within an algorithm-agile encryptor, may have a different latency, or per-cell encryption time. This differing latency may reorder cells, between VCs, and hence cause CDV. Algorithm-agile encryption preserves cell-order within an individual VC and also amongst VCs that use the same encryption algorithm. However, it may re-order cells that use different encryption algorithms. For example, let the cells from VCs 1 and 2 use encryption algorithms 1 and 2, respectively. Let algorithm 1 have a longer encryption time, per cell, than algorithm 2; then, for example, an input cell-sequence of $1,1,2,2, \ldots$ might produce the output cell-sequence $2,2,1,1, \ldots$. This introduces CDV into the output cell-stream. Both the single, shared-processor architecture and the multiple, parallel-processor architecture can generate cell reordering, and hence CDV. The interaction between this encryption-induced CDV and the ATM traffic-management policies can cause added cell-loss. Hence, an algorithm-agile encryptor should participate in the QoS negotiations during ATM callsetup. This participation requires models for the encryption-induced CDV.

Section 2, of this paper, proposes an architecture for an algorithm-agile encryptor that consists of $M$ parallel-pipelines that feed a common output-queue for the outgoing ATM-link (see Figure 1). Each pipeline is a keyagile encryptor that provides a single encryption algorithm for many VCs. Section 3 then bounds the CDV generated by this proposed architecture. It also explores the tradeoffs between CTD and CDV for various output-queuing algorithms (FIFO, priority and oldest-job-first). For FIFO output-queueing, the execution time for the longest pipeline bounds the CDV. For priority queuing, the CDV can be unbounded if there are three, or more, priority classes.

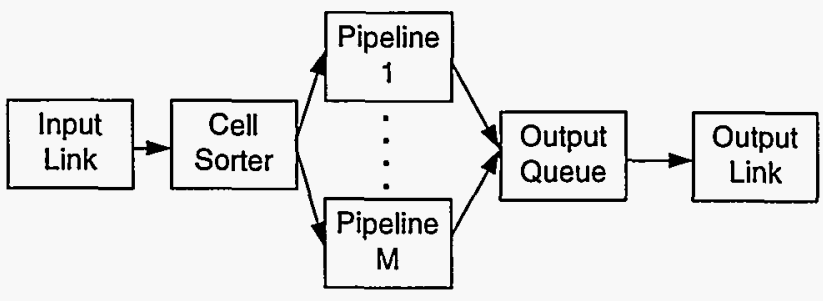

Figure 1: Parallel Pipeline Architecture for AlgorithmAgile Encryptor

\subsection{PARALLEL-PIPELINE ARCHITECTURE for ALGORITHM-AGILE ENCRYPTION}

Algorithm-agile encryptors come in two basic varieties -- namely a single shared-processor or multiple parallel-processors. In both cases, the encryptor serves one input ATM-link and one output ATM-link. So, the encryptor is single-input, single-output (SISO). Performance analysis for the single shared-processor case is a classic scheduling-problem. However, a single, shared-processor architecture may not scale to Gb/s ATM link-rates because of the context-switching overhead between different encryption algorithms. So, this paper considers the multiple parallel-processor architecture shown in Figure 1. As previously mentioned, this parallelarchitecture may trade increased scalability for some callblocking. Section 2.1 describes this generic architecture in greater detail. Section 2.2 briefly compares this encryptor architecture with ATM-switch designs.

\subsection{Components:}

In Figure 1, the Input Link extracts the incoming ATM cells from the incoming physical layer. It also performs the Header Error Check (HEC) function. It does not perform a Segmentation and Reassembly (SAR) function since the ATM Security Specification [1] uses per-cell encryption. The Output Link represents the corresponding functions for the outgoing ATM link.

The Cell Sorter assigns incoming cells to the correct pipeline (i.e., encryption algorithm). During callsetup, the Cell-Sorter might load the association between a $\mathrm{VC}$ and its encryption pipeline into a table-structure. These table-lookups are another potential bottleneck at $\mathrm{Gb} / \mathrm{s}$ rates. The design considerations include flat-memory versus associative memory and cache sizes. This paper does not explore these issues further. 
Sholander: The Effect of Algorithm-Agile Encryption on ATM Quality of Service

The $M$ pipelines represent $M$ different parallelprocessors. Each pipeline, $P_{i}$, implements a different encryption algorithm, $A_{i}$. Every VC that uses encryption algorithm $A_{i}$ shares Pipeline $P_{i}$. (Note: define the ATM cells that use $P_{i}$ as "type $i$ " cells.) So, each individual pipeline must be a key-agile encryptor. Since few encryption algorithms can encrypt an entire ATM-cell (384 payload bits) within one ATM-cell interarrival-time (about $2.8 \mu \mathrm{s}$ for transport in $155.52 \mathrm{Mb} / \mathrm{s}$ SONET OC$3 c$ ), each encryption pipeline might use $S_{i}$ stages. Each stage, $P_{i k}$, implements a portion of algorithm $A_{i}$ on each ATM-cell. (Section 3.2 discusses the general case where the encryption-block size is not equal to 384 bits, or one ATM cell). Section 3 discusses constraints on execution speed versus pipeline depth in greater detail. These constraints yield upper bounds on the CDV generated by this algorithm-agile encryptor.

The encryption pipelines may have different execution times. Hence, output-queueing is required. For example, let cells for VCs 1 and 2 use pipelines 1 and 2, respectively. Let algorithms 1 and 2 have normalized (to the ATM-cell interarrival-time) encryption times of 2 and 1 , respectively. Then, for example, an input cell-sequence of $1,2,1,2, \ldots$, at normalized times $0,1,2,3, \ldots$, has simultaneous arrivals at the output queue at (normalized) times 2 and 4 . The output-queueing discipline can be quite general. Section 3 gives upper bounds on the CDV generated by the FIFO, priority and oldest-job-first queueing disciplines.

Finally, this generic architecture glosses over the control issues associated with swapping $\mathrm{N}$ algorithms between $M$ pipelines. However, that swapping occurs over the time scale of VC call-setup requests rather than at the ATM-cell rate. So, the performance issues are blocking and added call-setup delay rather than context-switching speed.

\subsection{Comparison with ATM Switch Design:}

Algorithm-agile encryptors present different design problems than typical ATM switches. First, ATM switches are usually multiple-input, multiple-output devices. For each transmission direction, an algorithmagile encryptor is only single-input, single-output (SISO). So, Call-Admission Control (CAC) should be simpler for the encryptor. Second, while both devices generate CDV, the mechanisms are different. ATM switches try to keep internal path-delays equal, since this reduces CDVgeneration. However, output-queueing still occurs in ATM switches since simultaneous cell-arrivals at different inputs may have the same output link. This outputqueueing causes CDV in ATM switches. Third, cell-loss in ATM switches typically occurs because of finite outputbuffer size. Conversely, like any SISO device, algorithmagile encryptors can have zero cell-loss.

\subsection{PERFORMANCE RESULTS}

This section bounds the CDV generated by the parallel pipeline architecture proposed in Section 2. It also explores the tradeoffs between CTD and CDV for various output-queuing algorithms (FIFO, priority and oldest-jobfirst).

\subsection{Assumptions:}

All subsections use the following seven assumptions. First, the ATM cell interarrival time is normalized to 1 . Second, the CDV, CTD and the service times of each pipeline stage are then normalized to that cell interarrival-time. Third, the input link is full (i.e., no idle or unassigned cells). Fourth, each encryption pipeline, $i$, has a fixed execution-time, $T_{i}$, per ATM cell. Fifth, the output and input links are synchronous. Sixth, the analysis ignores the jitter caused by extracting/inserting ATM cells from/into the physical layer. Finally, the encryptor has zero cell-loss (i.e., each encryption pipeline can "keep up" with the full input cell-rate).

Assumptions one and two are for convenience. Assumptions three and four allow analytic tractability. In practice, a pipeline's per-cell execution-time may vary because of the context-switching between different VC's encryption-contexts. Assumptions five and six are reasonable if the physical layer is SONET. In any case, the physical-layer jitter should statistically affect all VCs identically. Finally, Section 3.2 derives a relationship between execution speed and pipeline depth that guarantees zero cell-loss. Other architectures where the individual encryption pipelines can't "keep up", may be studied in future work.

\subsection{Execution Speed and Cell Storage vs. Pipeline Depth:}

Consider the simplest "algorithm-agile" encryptor, which has one pipeline with one stage. That encryptor is a simple $D / D / 1$ queue with service time $T_{1}$. Stability, and hence no cell-loss, of course requires $T_{1} \leq 1$.

A slightly more complex system is one pipeline with $S$ stages. Let each stage have a service-time of $T_{1 j}$ where:

$$
\mathrm{T}_{1}=\sum_{\mathrm{j}=1}^{\mathrm{S}} \mathrm{T}_{\mathrm{lj}}
$$


Let $I_{k}$ and $O_{k}$, for $k=0,1,2,3, \ldots$, be the input and output times for the $\mathrm{k}^{\text {th }}$ input-cell. In this case, stability occurs if $\mathrm{O}_{\mathrm{k}} \leq \mathrm{I}_{\mathrm{k}+\mathrm{S}}$. This implies that $\mathrm{T}_{1} \leq \mathrm{S}$. So, an individual stage can have a $T_{1 j}>1$-- as long as the overall sum is still less than, or equal to, $\mathrm{S}$. A final simple result is that this single pipeline can't store more than $S$ cells, since that is the maximum number of arrivals during $S$ unit interarrivaltimes. (Note: if the encryption-block size is not equal to 384 bits, or one ATM cell, then the physical hardware model should be mapped into this paper's general logical model. For example, a pipe-lined version of DES (Data Encryption Standard) [5] might use 16 physical stages where each physical stage implements one of the 16 DES rounds. Since DES has an encryption block-size of 64-bits, an ATM-cell would be split between several rounds. This physical implementation would then correspond to $T_{1}=$ $8 / 3$ and $S=3$ if each DES round takes $1 / 6$ normalized time units).

Now consider multiple pipelines, $P_{\mathrm{i}}$. Let each pipeline have $S_{i}$ stages. Then stability requires $T_{i} \leq S_{i}$, for all i. As a collorary, the overall encryptor can't store more $S_{k}$ cells total, where $S_{k}$ is the largest of the $S_{i}$ 's, in its pipelines and output queue. (For both claims, just assume various input cell-streams of all type $i$ cells.) The next section uses these simple results to derive bounds on both the maximum CDV and the worst-case CDV probabilitydensity.

\subsection{Bounds:}

\subsubsection{Conditions for No CDV Generation:}

Consider, the two pipeline case. Let the two pipelines have execution times of $T_{1}$ and $T_{2}$, respectively. Let cells $k$ and $k+1$ be of types 1 and 2, respectively. Cellorder is clearly maintained if $\left(\mathrm{T}_{1}-\mathrm{T}_{2}\right)<1$. Reversing the cell input-order shows that cell-order is maintained whenever $\left|T_{1}-T_{2}\right|<1$. For a general number of pipelines, consider pair-wise arrival patterns. This leads to the general rule. The proposed parallel-pipeline architecture does not generate CDV if:

$$
\left|T_{i}-T_{j}\right|<1, \text { for all } i, j
$$

\subsubsection{Bounds for FIFO Output Queueing:}

The previous equation will probably not hold for most algorithm-agile encryptors. So, consider a twopipeline system where $T_{1} \geq T_{2}+1$. Also assume FIFO (First-In, First-Out) output queueing. Let the arriving cell be type 1. From the stability results given in Section 3.2, that arriving type 1 cell can neither pass nor be delayed by previously arrived type 1 or type 2 cells. So, that type 1 cell's CDV depends on how many later-arriving type 2 cells pass it. If a type 1 cell arrives at time $t=k$, then it can be passed by type 2 cells that arrive in the interval $C$.

$$
\begin{aligned}
& \mathrm{C}=\left\{\mathrm{k}+1, \mathrm{k}+2, \ldots, \mathrm{k}+\left\lfloor\mathrm{T}_{1}-\mathrm{T}_{2}\right\rfloor\right\}, \\
& \text { where }\lfloor\mathrm{x}\rfloor \text { is the largest integer } \leq \mathrm{x}
\end{aligned}
$$

Letting $\mathrm{T}_{2}$ go to zero, yields another useful result. The CDV for type 1 cells is bounded by the largest integer $\leq$ $T_{1}$. (The CDV for type 2 cells is discussed below.) This result generalizes to multiple pipelines if $T_{1}$ denotes the execution time for the longest pipeline.

For the two-pipeline case, the CDV probabilitydensity for type 1 cells is the probability of $x$ type 2 cellarrivals in the interval C. Assume I.I.D. (Independent, Identically Distributed) arrivals with probabilities $\alpha_{1}$ and $\alpha_{2}$ for type 1 and type 2 cells, respectively. (Positivelycorrelated arrivals produce less CDV than I.I.D. arrivals.) Then, the CDV probability-density for type 1 cells has a binomial distribution.

$$
\begin{gathered}
\operatorname{Pr}(\text { Type 1's CDV }=\mathrm{x} \text { cells })=\left(\begin{array}{l}
\Delta \\
\mathrm{x}
\end{array}\right) \alpha_{2}^{\mathrm{x}} \alpha_{1}^{\Delta-\mathrm{x}}, \\
\text { where } \Delta=\left\lfloor\mathrm{T}_{1}-\mathrm{T}_{2}\right\rfloor
\end{gathered}
$$

For a full input-link, previously-stored type 1 cells cause the CDV experienced by type 2 cells. Hence, both cell types have identical CDV probability-densities. (Note: this is not true if the input-link contains idle, or unassigned, cells.) In the general case, for a full input-link, an induction argument shows that the CDV probabilitydensities are identical for all cell types. (Again, this is not true if the input-link contains idle, or unassigned, cells.)

For a general number of pipelines, the worst-case CDV probability-density entails sums of multinomial terms. For example, consider the three-pipeline case. Assume an interesting case, such as $T_{1} \geq T_{2}+1$ and $T_{2} \geq$ $\mathrm{T}_{3}+1$. Let $\Delta_{2}=\left\lfloor\mathrm{T}_{1}-\mathrm{T}_{2}\right\rfloor$ and $\Delta_{3}=\left\lfloor\mathrm{T}_{1}-\mathrm{T}_{3}\right\rfloor$. Then, define the interval $\mathrm{D}$ as follows:

$$
\mathrm{D}=\left\{\mathrm{k}+1, \mathrm{k}+2, \ldots, \Delta_{3}\right\}
$$

Then the probability that the $\mathrm{CDV}$, for an arriving type 1 cell, is $\mathrm{x}$ cells is:

$$
\begin{gathered}
=\sum_{y=0}^{x} \operatorname{Pr}(y \text { type } 3 \text { cells in the interval }(D-C) \\
\text { and }(x-y) \text { type } 2 \text { or } 3 \text { cells in } C)
\end{gathered}
$$


Sholander: The Effect of Algorithm-Agile Encryption on ATM Quality of Service

$$
\begin{aligned}
& =\sum_{\mathrm{y}=0}^{\mathrm{x}}\left\{\left(\begin{array}{c}
\Delta_{3}-\Delta_{2} \\
\mathrm{y}
\end{array}\right) \alpha_{3}^{\mathrm{y}}\left(\alpha_{1}+\alpha_{2}\right)^{\Delta_{3}-\Delta_{2}-\mathrm{y}}\right\} * \\
& \left\{\left(\begin{array}{c}
\Delta_{2} \\
x-y
\end{array}\right)\left(\alpha_{2}+\alpha_{3}\right)^{x-y}\left(\alpha_{1}\right)^{\Delta_{2}-(x-y)}\right\}
\end{aligned}
$$

The intervals $\mathrm{C}$ and $\mathrm{D}$ are defined above. The I.I.D. arrival probabilities are again $\alpha_{1}, \alpha_{2}$ and $\alpha_{3}$ for types 1,2 and 3, respectively. (Note: the sums exclude terms where $y>\left(\Delta_{3}\right.$ $\left.-\Delta_{2}\right)$ or $\left.(\mathrm{x}-\mathrm{y})>\Delta_{2}\right)$.

The general case is straightforward, but messy. However, algorithm-agile encryptors probably have a small number of parallel pipelines (e.g., $\leq 5$ ). So, the CDV probability-density equations remain tractable.

\subsubsection{Priority Output Queueing:}

Consider the two-pipeline case with $\mathrm{T}_{1} \geq \mathrm{T}_{2}+1$. However, let type 1 cells have priority in the output queue. Then, every type 1 cell has a CTD of $\left\lceil\mathrm{T}_{1}\right\rceil+1$ and a CDV of 0 . For type 2 cells, again assume a full input-link. Then, in steady-state, each arriving type 2 cell sees exactly $\left\lceil\mathrm{T}_{1}+1\right\rceil$ cells in front of it. By $\mathrm{D} / \mathrm{D} / 1$ stability, each of those cells must depart before that arriving type 2 cell. (The type 1 have priority. The type 2 cell can't pass other type 2 cells since the output queueing discipline is still FIFO within a priority class.) So, both cell-types have the same CTD and CDV.

Now consider the three-pipeline case. Assume that $T_{1} \geq T_{2}+1$ and $T_{2} \geq T_{3}+1$. Let the output-queue prioritylevels be type1, type 2 and type 3 . Since type 1 cells have priority, the CTD and CDV are identical to the twopipeline case. Type 2 cells may have less CTD, but more $\mathrm{CDV}$, than in the two-pipeline case. If an arriving type 2 cell sees only type 1 and type 2 cells in the system then its CTD is $\left\lceil T_{1}+1\right\rceil$. Alternatively, if an arriving type 2 cell sees only type 2 and type 3 cells in the system then its CTD is $\left\lceil\mathrm{T}_{2}+1\right\rceil$. So, the type $2 \mathrm{CDV}$ is bounded by $\left\lceil\mathrm{T}_{1}+1\right\rceil-\left\lceil\mathrm{T}_{2}+1\right\rceil=\left\lceil\mathrm{T}_{1}-\mathrm{T}_{2}\right\rceil$. However, type 3 cells do not have bounded CDV and CTD. For example, let $\mathrm{T}_{1}$ equal $3, T_{2}$ equal 2 and $T_{3}$ equal 1 . Let the input cellsequence be $\{1,1,1,3,2,2,2,2, \ldots\}$. Then, the type 3 cell never leaves the output queue.

\subsubsection{Oldest-Job-First Output Queuing:}

Priority queueing can yield unstable behavior if there are three, or more, priority classes. An alternative queueing discipline is Oldest-Job-First (OJF). This discipline transmits, from the output queue, whichever cell has been in the system longest. Hence, OJF gives preference to cells from the pipeline with the longest execution-time. Again, assume a full input-link. Let $T_{1}$ be the execution time for the longest-time pipeline. In that case, OJF yields the same CTD, namely $\left\lceil\mathrm{T}_{1}+1\right\rceil$, and zero CDV for all cell-types for any number of pipelines. (Again, if the input link contains idle cells then each celltype may still have a different CTD and non-zero CDV).

\subsection{CONCLUSIONS:}

This paper discussed the differences between keyagile ATM encryptors [2,3] and proposed algorithm-agile ATM encryptors. An algorithm-agile encryption architecture, that used multiple, parallel encryptionpipelines, was proposed. That algorithm-agile encryptor's effect on the ATM Quality of Service (QoS) metrics, such as Cell Transfer Delay (CTD) and Cell Delay Variation (CDV), was analyzed. Bounds on the maximum CDV and the CDV's probability density were derived. The key result was that pipelined algorithm-agile encryptors could cause CDV even if the constituent encryption-pipelines kept up with the input ATM cell-rate.

One solution appends delay-lines to each encryption pipeline, such that the inequality in Section 3.3.1 holds. In that case, the encryptor can trade zero CDV for increased CTD. For example, an algorithm-agile encryptor that implements this delay-line technique for null-encryption, DES and Triple-DES [5] could have a CTD of 8 ATM cell interarrival-times, which is about 21 $\mu s$ at the SONET OC-3 rate. However, this simple delayline solution can complicate adding new algorithms to an existing algorithm-agile encryptor.

\section{REFERENCES:}

[1] Phase 1 ATM Security Specification (Draft), ATM Forum BTD-Security-01.00, December 1996.

[2] D. Stevenson, N. Hillery and G. Byrd, "Secure Communications in ATM Networks", Communications of the ACM, pp. 45-52, February, 1995.

[3] C. Wilcox, "ATDNet Research at the National Security Agency", IEEE Network, pp. 42-47, July/August, 1996.

[4] Traffic Management Specification Version 4.0, ATM Forum af-tm-0056.000, April 1996.

[5] B. Schneier, Applied Cryptography, Second Edition, John Wiley, New York, 1996. 